\title{
Transient Intracranial Circulatory Arrest Evidenced at the Time of Intracranial Aneurysm Rupture: Case Report
}

\author{
Ilari Rautalin * (1) and Miikka Korja
}

() 2020 The Author(s)

\section{Introduction}

One-fourth of people with aneurysmal subarachnoid hemorrhage (SAH) die from the bleed suddenly in emergency rooms or before reaching hospital [1]. Unless medicolegal or medical autopsies are systematically performed to all sudden deaths, these SAH deaths are almost invariably classified as sudden cardiac deaths. The first observable symptom of sudden SAH death is loss of consciousness (LOC), which is also a common presenting symptom of non-fatal SAH. The pathophysiology of LOC at the time of aneurysm rupture is controversial. However, similar to sudden SAH deaths, the etiology of LOC in non-fatal SAHs is often considered to be cardiogenic. This presumption may, however, stem from the lack of evidence suggesting other etiologies.

This report illustrates that a transient intracranial circulatory arrest associates with LOC and can occur without cardiac arrest. Furthermore, the case shows that patients experiencing a transient intracranial circulatory arrest can recover relatively well, despite the poor clinical status at the time of bleed.

\section{Clinical Presentation}

Reporting follows the CARE guideline [2]. According to the Finnish legislation, the patient consent is not required to use de-identified patient information for scientific purposes.

\footnotetext{
*Correspondence: ilari.rautalin@helsinki.fi

Department of Neurosurgery, University of Helsinki and Helsinki

University Hospital, Topeliuksenkatu 5, P.O. Box 266, 00029 Helsinki, Finland
}

\section{Presenting Symptoms}

A 50- to 60-year-old ex-smoker with hypertension and asthma lost consciousness while playing sports. Bystanders performed cardiopulmonary resuscitation, and the patient regained consciousness in a couple of minutes.

\section{Clinical Findings}

On admission, the patient was alert and neurologically intact but hypertensive and suffered from severe headache. While positioning for a head computed tomography $(\mathrm{CT})$ scan, the patient became unresponsive and had sinus bradycardia (35 beats per minute). Pupils were still normal. Following intubation on the CT scan table, a head CT scan and a subsequent CT angiography (CTA) with an automated contrast injector system was performed. During CTA, systolic blood pressure raised suddenly over $200 \mathrm{mmHg}$ and both pupils became nonreactive and dilated, despite sedation and antihypertensive medication. The CTA scan showed intracranial circulatory arrest at the level of proximal middle and anterior cerebral arteries (Fig. 1a, Supplementary Video 1). Extracranial arteries filled normally (Fig. 1a, Supplementary Video 1), confirming that CTA was performed properly. CT revealed diffuse subarachnoid hemorrhage (SAH) and a small intracerebral hematoma but no intracranial aneurysms (IAs). The patient was given a rapid infusion of mannitol, and the pupils normalized within $10 \mathrm{~min}$. After stabilizing the patient, a follow-up CTA was performed $57 \mathrm{~min}$ after the first one. The scan showed a regained intracranial circulation (Fig. 1b, Supplementary Video 2), signs of a rebleed (Fig. 1b, Supplementary Video 2), and a suspicious lesion at the level of a right posterior communicating artery. In a following conventional catheter angiography one hour later, a right posterior communicating artery aneurysm was clearly

\section{望 Springer}



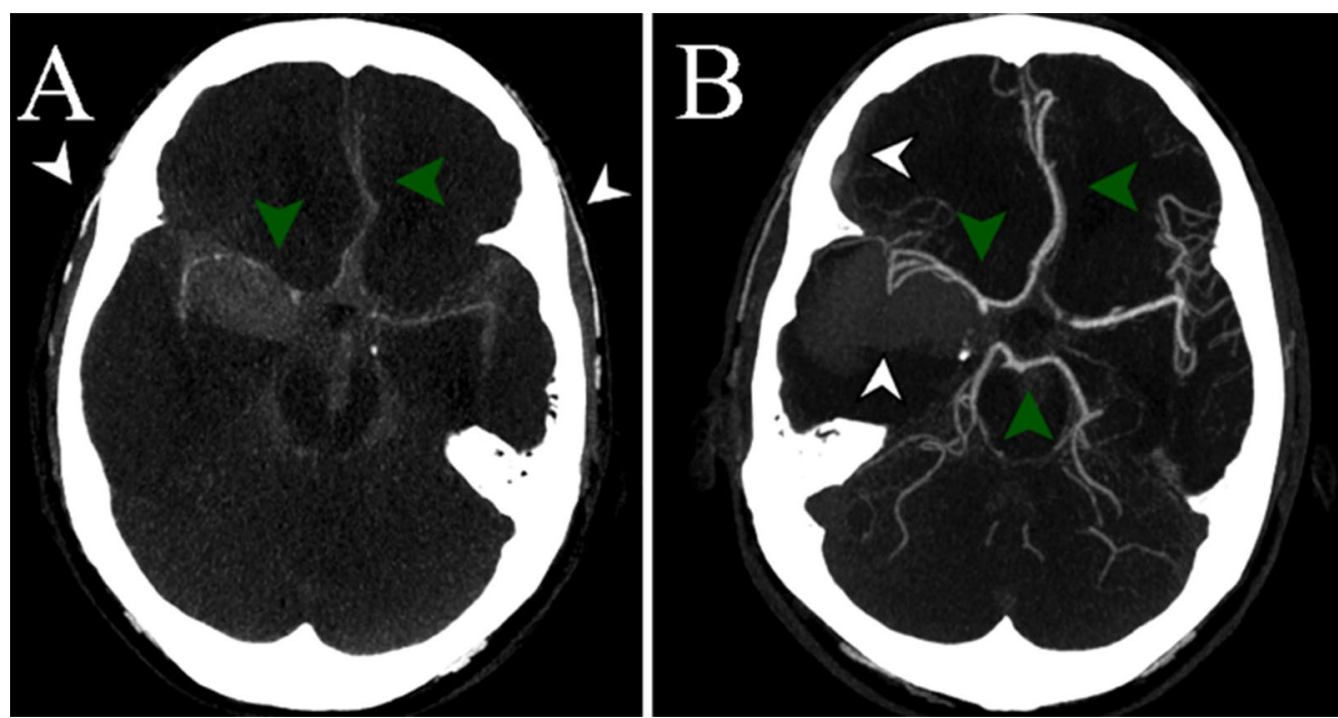

Fig. 1 Computed tomography angiography (CTA) scans. At the time of aneurysm rupture and intracranial circulatory arrest (a), anterior and middle cerebral arteries filled vaguely (a; green arrowheads) whereas extracranial temporal arteries were clearly viewed (a; white arrowheads). After regaining intracranial circulation (b), anterior, middle and posterior cerebral arteries were filling normally again (b; green arrowheads). CTA showed also signs of a rebleed, such as a grown intracerebral hematoma and a new right acute subdural hematoma (b; white arrowheads). Computed tomography scan confirmed the rebleed (images not shown) (Color figure online)

visible (Fig. 2a). Based on a 12-lead electrocardiogram and biomarkers, no signs of stress cardiomyopathy (i.e., takotsubo cardiomyopathy) were observed during the hospitalization period.

\section{Treatment and Outcome}

Following angiography, the aneurysm was surgically clipped (Fig. 2b), and intracerebral and subdural hematomas were evacuated. Later, the patient developed hydrocephalus, which was treated with a ventriculoperitoneal shunt. The patient returned to work (higher education) one year after SAH, but due to neurocognitive sequelae, further rehabilitation was necessary. However, the patient was fully independent, lived at home, and continued sport activities, such as recreational running.
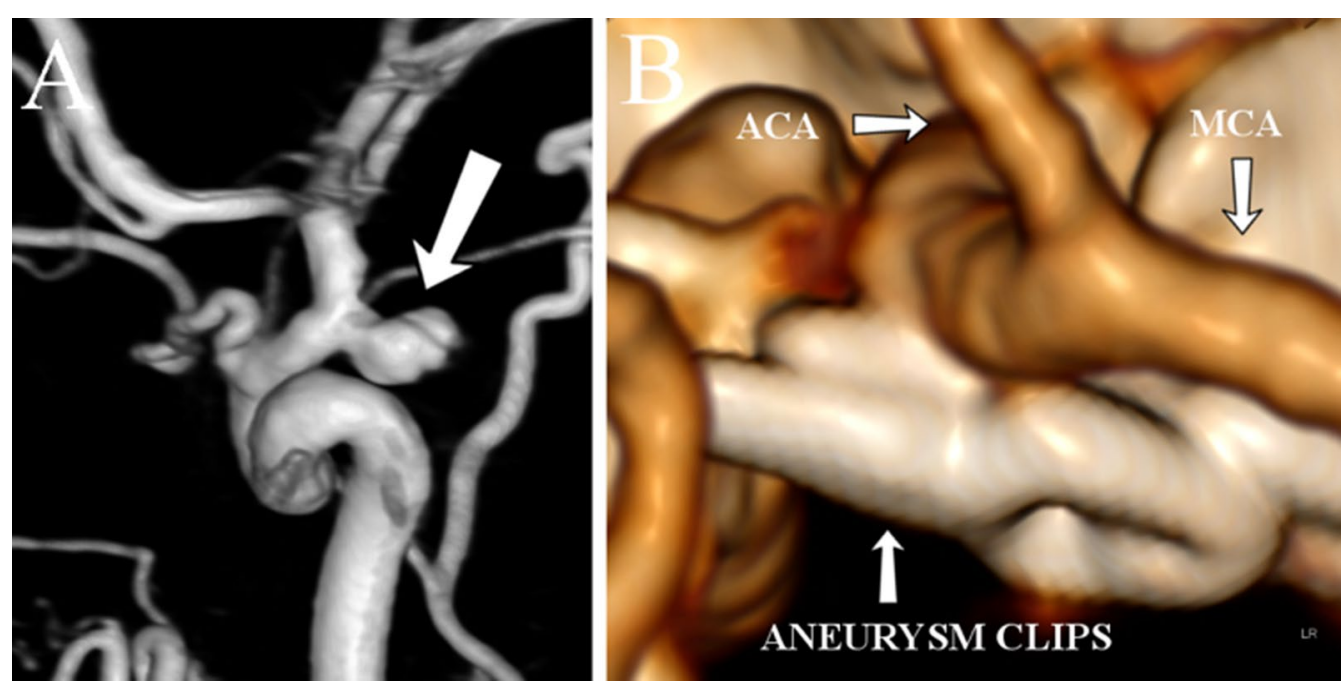

Fig. 2 A conventional catheter angiography showed a right posterior communicating artery aneurysm (a; white arrow). After surgery, computed tomography angiography demonstrated that the aneurysm was clipped (b). MCA = middle cerebral artery, ACA=anterior cerebral artery (Color figure online) 


\section{Discussion}

Sudden SAH death manifests as LOC and dilatation of pupils, and at least $40 \%$ of hospitalized SAH patients experience abnormal alteration of consciousness at the onset of SAH [3]. Fortunately, LOC is transient in many SAH patients [3]. Furthermore, a vast increase in the intracranial pressure (ICP) at the time of LOC is likely one of the major events that contribute to the beneficial formation of intra- and extraluminal thrombi, which aid to stop the bleeding from the ruptured aneurysm. In the presented case, the early CTA scan demonstrated the regained intracranial circulation (Fig. 1b, Supplementary Video 2) and a suspicious posterior communicating aneurysm, which most likely was partially thrombosed at the time of imaging.

Pathogenetic phenomena explaining transient LOC and pupillary dilatation in humans at the time of aneurysmal SAH have been speculated for a long time but never evidenced by CTA imaging. In the 1970s, Nornes and Magnaes investigated the dynamics of ICP in SAH patients during rebleedings and showed that an IA rupture may lead to ICP levels that exceed the diastolic blood pressure $[4,5]$. Moreover, Nornes showed that circulation in the internal carotid artery stops at the end of diastole in aneurysmal SAH patients, most likely due to a rise in ICP [5]. These reports $[4,5]$ provided a better explanation for new hypotheses about intracranial events at the time of SAH. According to one widely accepted hypothesis, blood jetting through a ruptured aneurysm causes a rapid increase in ICP that in turn leads to pupillary dilatation. When ICP reaches the level of mean arterial pressure, cerebral perfusion pressure approaches zero, and intracranial circulatory arrest is presumed to happen. This is, however, the very first time that true but transient arrest of cerebral circulation has been documented.

In short, the presented images and clinical cascade document that at the time of aneurysm rupture, intracranial circulatory arrest happens simultaneously with pupillary dilatation and LOC. LOC increases the risk of poor outcome after SAH nearly threefold [3], but good recovery is evidently possible.

\section{Conclusion}

Transient LOC in aneurysmal SAH patients is likely to be caused by a transient intracranial circulatory arrest, as has been speculated for a long time. In the described case, LOC did not accompany with transient cardiac arrest. Whether such simultaneous phenomenon occasionally happens remains to be documented.

\section{Electronic supplementary material}

The online version of this article (https://doi.org/10.1007/s12028-020-00948 -w) contains supplementary material, which is available to authorized users.

\section{Acknowledgements}

Open access funding provided by University of Helsinki including Helsinki University Hospital.

\section{Conflict of interest}

The authors declare that they have no conflicts of interest.

\section{Ethical approval}

Our case study has been written following the principles of the Declaration of Helsinki. According to the Finnish legislation, the patient consent is not required to use de-identified patient information for scientific purposes. Reporting follows the CARE guideline.

\section{Open Access}

This article is licensed under a Creative Commons Attribution 4.0 International License, which permits use, sharing, adaptation, distribution and reproduction in any medium or format, as long as you give appropriate credit to the original author(s) and the source, provide a link to the Creative Commons licence, and indicate if changes were made. The images or other third party material in this article are included in the article's Creative Commons licence, unless indicated otherwise in a credit line to the material. If material is not included in the article's Creative Commons licence and your intended use is not permitted by statutory regulation or exceeds the permitted use, you will need to obtain permission directly from the copyright holder. To view a copy of this licence, visit http://creativecommons.org/licenses/by/4.0/.

\section{Publisher's Note}

Springer Nature remains neutral with regard to jurisdictional claims in published maps and institutional affiliations.

Published online: 26 March 2020

\section{References}

1. Korja M, Lehto H, Juvela S, Kaprio J. Incidence of subarachnoid hemorrhage is decreasing together with decreasing smoking rates. Neurology. 2016:87:1118-23.

2. Gagnier JJ, Kienle G, Altman DG, et al. The CARE guidelines: consensusbased clinical case report guideline development. J Clin Epidemiol. 2014;67:46-51

3. Suwatcharangkoon S, Meyers E, Falo C, et al. Loss of consciousness at onset of subarachnoid hemorrhage as an important marker of early brain injury. JAMA Neurol. 2016;73:28-35.

4. Nornes H, Magnaes B. Intracranial pressure in patients with ruptured saccular aneurysm. J Neurosurg. 1972;36:537-47.

5. Nornes $\mathrm{H}$. The role of intracranial pressure in the arrest of hemorrhage in patients with ruptured intracranial aneurysm. J Neurosurg. 1973:39:226-34. 